\title{
Penfigoide cicatricial, causa poco común de estenosis supraglótica
}

\section{Cicatricial pemphigoid: Uncommon cause of supraglottic stenosis}

\author{
Héctor Bahamonde $\mathrm{S}^{1}$, Paul H. Délano R¹, Nicolás Albertz $\mathrm{A}^{2}$, Constanza Valdés $\mathrm{P}^{1}$.
}

\begin{abstract}
RESUMEN
日 penfigoide cicatricial o de mucosas es una patología que afecta principalmente a la mucosa oral y ocular. Sólo en $25 \%$ de los pacientes afecta también la piel y en alrededor de $10 \%$ de los pacientes compromete a la mucosa faringo-laríngea, produciendo en los casos severos disnea que incluso puede requerir traqueostomía En el tratamiento del penfigoide cicatricial habitualmente se utilizan corticoides $e$ inmunosupresores. En este artículo presentamos un caso de una mujer de 69 años con diagnóstico de penfigoide cicatricial que se manifiesta con disfagia y se comprueba con nasofibroscopía una estenosis supraglótica. Se discute el diagnóstico y manejo de esta patología poco habitual.
\end{abstract}

Palabras clave: Penfigoide, estenosis supraglótica, úlceras orales, disnea, disfagia.

\section{ABSTRACT}

Cicatricial or mucous membranes pemphigoid is a rare pathology that affects mainly the ocular and oral mucosae. In only $25 \%$ of patients the skin is affected, and in $10 \%$, the pharyngolaryngeal mucosa, resulting in serious cases in dyspnea that could even require a tracheostomy. Treatment is usually based on corticoids and immunosupressors. In this article we present the case of a 69 year old woman diagnosed with cicatricial pemphigoid, that presented dysphagia. A nasoendoscopy revealed supraglottic stenosis. Diagnosis and treatment of this uncommon disease are discussed.

Key words: Pemphigoid, supraglottic stenosis, oral ulcer, dyspnea, dysphagia.

Médico Servicio de Otorrinolaringología, Hospital Clínico Universidad de Chile.

2 Médico Cirujano, Universidad de Chile. 


\section{INTRODUCCIÓN}

Los penfigoides corresponden a un grupo especial de enfermedades autoinmunes caracterizadas por la formación de vesículas y ampollas subepidérmicas o submucosas causadas por la síntesis de autoanticuerpos contra elementos de la membrana basal de epidermis o mucosas ${ }^{1,2}$. Los penfigoides se clasifican en (i) penfigoide buloso que compromete primariamente la piel, y (ii) penfigoide cicatricial o penfigoide de las membranas mucosas. Esta última patología se caracteriza por ser una enfermedad vesículo ampollosa crónica, localizada y progresiva con predilección por membranas mucosas y afectación cutánea sólo de manera ocasional' ${ }^{1}$.

El penfigoide cicatricial fue descrito por primera vez por Cooper en $1857^{3}$. Clínicamente se manifiesta por vesículas 0 ampollas con rápida evolución hacia erosiones, teniendo como característica distintiva la formación de cicatrices residuales, que son las que terminan produciendo las complicaciones propias de esta patología, como pueden ser ceguera por compromiso ocular 0 incluso la muerte por afectación laríngea resultando en una obstrucción respiratoria ${ }^{4-6}$. Las zonas más afectadas son la mucosa oral, manifestándose como gingivitis descamativa crónica, y la mucosa conjuntival originando erosiones que llevan a la aparición de sinequias que incluso pueden conducir a la ceguera. Sin embargo también se puede ver compromiso de fosas nasales, faringe, laringe, esófago, uretra, vagina y ano $0^{2,7,8}$.

La edad más habitual de presentación es alrededor de los 60 años, la mayoría entre los 40 y los 80. Es más frecuente en mujeres en una proporción de dos a uno con respecto a los hombres. No hay predilección racial 0 geográfica ${ }^{4,6,7}$. En general es una enfermedad poco frecuente con incidencias anuales entre 2 a 5 por cada 100.000 habitantes 9 .

El diagnóstico es resultado de la historia clínica en conjunto con elementos histológicos e inmunopatológicos, que permitan demostrar la presencia de la enfermedad ${ }^{7}$. Se puede apreciar inflamación crónica en el tejido conectivo. En la inmunofluorescencia directa se observan depósitos lineales de IgG y factor 3 del complemento en las membranas basales del epitelio perilesional. Además se pueden encontrar depósitos de IgA e
IgM. En la inmunofluorescencia indirecta (IFI) se detectan IgG anti-membrana basal circulantes en el plasma. Utilizando técnicas como la IFI sobre piel dividida con cloruro sódico, se aumenta la sensibilidad permitiendo la distinción entre aquellos antígenos que están localizados en el lado epitelial de los localizados en el lado dérmico. El antígeno más comúnmente encontrado es una proteína de hemidesmosoma conocida como BPAg-2 o BP180. Los pacientes con penfigoide cicatricial también pueden poseer anticuerpos circulantes contra cerca de 10 antígenos diferentes de la membrana basal como: laminina 5 , colágeno VII, alfa-integrina, BPAg-1, entre otros ${ }^{4,5}$.

Por ser una enfermedad inmunológica que afecta a diversos tejidos, su diagnóstico y tratamiento debe ser multidisciplinario. Por esto, los pacientes deben ser evaluados idealmente por dermatología, oftalmología, otorrinolaringología y odontología.

A continuación se presenta el caso clínico de una paciente atendida en policlínico de Otorrinolaringología del Hospital Clínico Universidad de Chile con compromiso faringo-laríngeo por penfigoide cicatricial.

\section{CASO CLINICO}

Consulta paciente de 69 años, sexo femenino, por cuadro de 3 años de evolución caracterizado por cuadro de disfagia ilógica, prurito ótico y faríngeo, asociado a la aparición de úlceras nasales y orales intermitentes. El cuadro además se acompañó de entropión, es decir inversión de los bordes del párpado inferior provocando el roce de las pestañas con la superficie ocular, que requirió tratamiento quirúrgico en oftalmología en 3 oportunidades con empeoramiento progresivo de éste. De forma concomitante la paciente refiere aparición de costras pruriginosas en cuero cabelludo. No presentaba alteraciones genitales ni otros antecedentes relevantes. Al examen físico inicial destaca la presencia en cuero cabelludo de una placa alopécica de aproximadamente 10 por $3 \mathrm{~mm}$ de base eritematosa con una costra en su centro (Figura 1). A nivel ocular un importante entropión, adherencia del párpado al globo ocular (simbléfaron), distorsión de la salida de las pesta- 


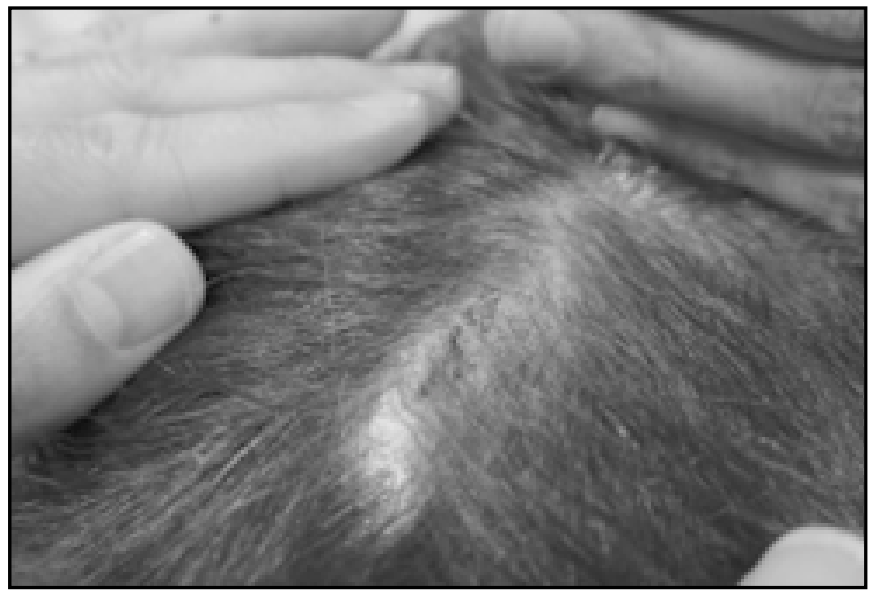

Figura 1. Lesión en cuero cabelludo producida por penfigoide cicatricial en mujer de 69 años.

ñas, edema e inyección conjuntival (Figura 2). En mucosa oral destacan erosiones de 2 centímetros de diámetro en paladar blando (Figura 3).

Dentro del estudio otorrinolaringológico se realiza nasofibroscopía laríngea que evidencia múltiples erosiones en paladar blando, faringe y estenosis supraglótica (Figuras 4 y 5). Se toman muestras para biopsia de mucosa bucal y velo del paladar en 2 oportunidades. La primera, de velo del paladar concluye proceso inflamatorio crónico activo ulcerado en mucosa oral. La segunda biopsia, tomada del paladar blando, se informa compatible con lesiones tipo penfigoide (Figura 6). Se realiza- ron inmunofluorescencia directa en piel e indirecta en sangre que resultaron negativas. Además se buscó la presencia de $p$ y cANCA resultando ambos negativos.

En evaluación por dermatología se miden anticuerpos antiepiteliales para penfigoide resultando $\lg A(+)$ 1/10, IgG e IgM negativos. Con estos hallazgos se diagnostica penfigoide cicatricial o penfigoide de membranas mucosas iniciándose tratamiento inmunosupresor con azatioprina y dapsona. La paciente evoluciona favorablemente con mejoría de su cuadro, manteniéndose en control por otorrinolaringología, dermatología y oftalmología.

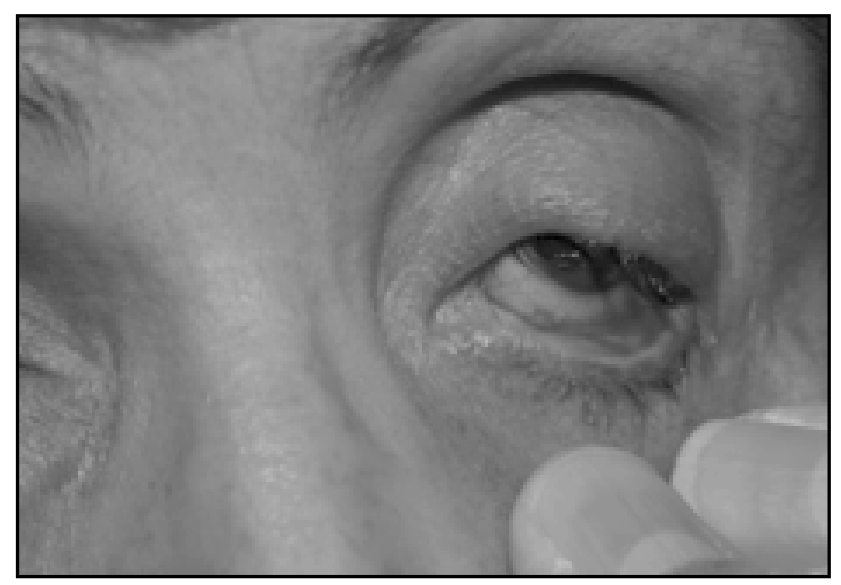

Figura 2. Entropión, Simbléfaron en paciente con diagnóstico de penfigoide cicatricial. 


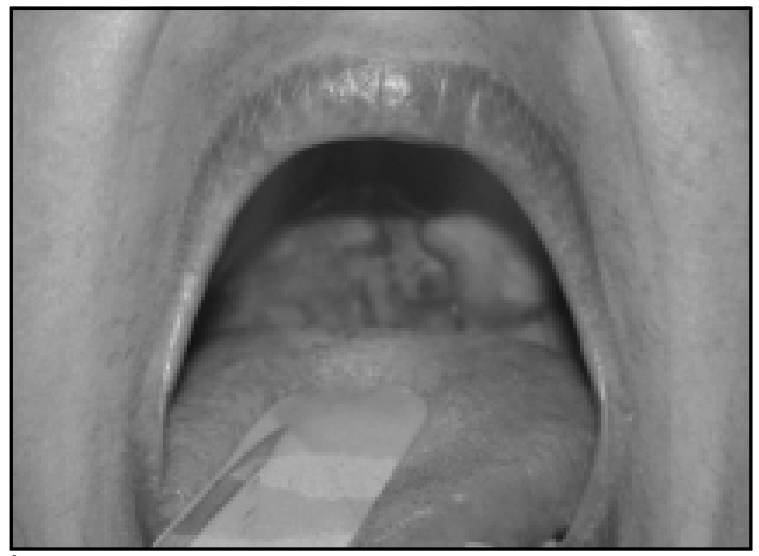

Figura 3. Úlceras en paladar blando en paciente con diagnóstico de penfigoide cicatricial.

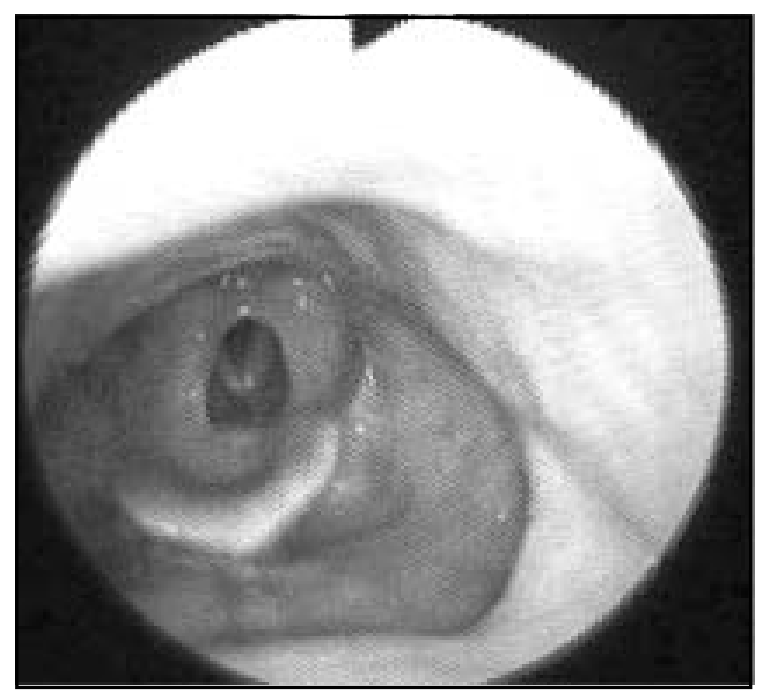

Figura 4. Imagen de laringe obtenida por nasofibroscopía en paciente con penfigoide cicatricial. Se observa membrana cicatricial tipo circunferencia supraglótica que produce una estenosis supraglótica.

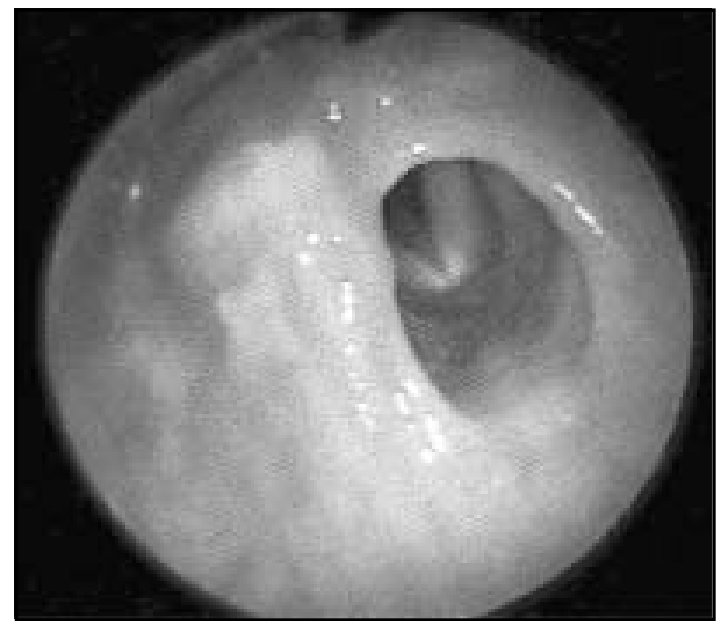

Figura 5. Imagen de glotis y supraglotis obtenida por nasofibroscopía en paciente con penfigoide cicatricial. Se observa membrana cicatricial tipo circunferencia supraglótica que no compromete a las cuerdas vocales. 


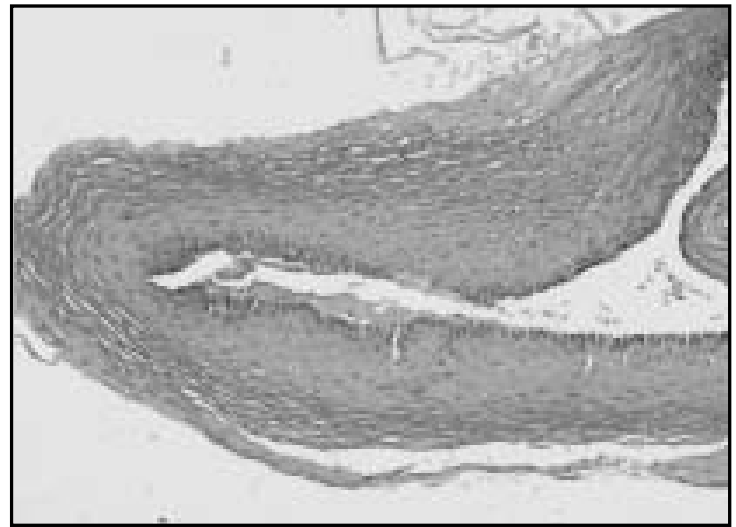

Figura 6. Corte histológico de biopsia tomada de la mucosa del paladar blando compatible con diagnóstico de penfigoide cicatricial de mucosas. Se observa lesión bulosa en mucosa.

\section{DISCUSIÓN}

\section{Diagnóstico del penfigoide cicatricial}

El principal diagnóstico diferencial del penfigoide cicatricial incluye al pénfigo, lupus eritematoso sistémico, liquen plano y eritema multiforme. Además de la historia clínica, el diagnóstico de confirmación se apoya en la inmunofluorescencia directa y en la biopsia ${ }^{5}$. La biopsia debe ser tomada de una vesícula 0 de tejido sano perilesional, pero no de una placa necrótica. La histología se caracteriza por la presencia de depósitos de IgG y fragmentos del complemento en la membrana basal y dermis. La inmunofluorescencia directa resulta positiva entre $80 \%$ a $97 \%$ de los pacientes, mientras que en los restantes el diagnóstico se basa en la clínica y la biopsia compatible con penfigoide cicatricial ${ }^{9}$. En nuestro paciente la IFI fue negativa, pero la historia clínica más la histología sustentan el diagnóstico de penfigoide cicatricial.

\section{Compromiso laríngeo y penfigoide cicatricial}

El penfigoide cicatricial es poco frecuente con incidencias anuales de 2 a 5 por cada 100.000 habitantes $^{10}$. Thost, en 1911 fue el primero en describir el compromiso laríngeo en el pénfigo cicatricial ${ }^{11}$. El compromiso laríngeo está presente en cerca del $10 \%$ de los pacientes con penfigoide cicatricial ${ }^{12}$. Hanson publicó una serie de 142 pacientes con penfigoide cicatricial y sólo 13 de éstos pacientes presentaba compromiso laríngeo, y de estos 13 pacientes sólo 3 presentaba algún grado de estenosis laríngea clínicamente significativa, lo que corresponde a cerca de $2 \%$ del total de pacientes con penfigoide cicatricial ${ }^{12}$. La paciente presentada en este artículo consultó por disfagia y al examen endoscópico presentaba un anillo estenótico a nivel de la supraglotis comparable a los 3 pacientes con estenosis laríngea clínicamente significativa descritos por Hanson en 1988. Boedecker publicó en el año 2003 un caso de estenosis laríngea severa por penfigoide cicatricial que presentaba, además del compromiso supraglótico, estenosis subglótica en el que se sugirió manejo con traqueostomía, pero que finalmente manejaron con corticoides e inmunosupresores ${ }^{4}$. Gaspar y Wojnarowska ${ }^{13}$ describen un caso de penfigoide cicatricial con compromiso laríngeo que requirió manejo con traqueostomía. Así mismo 0jha y colaboradores, describen el caso de una mujer de 18 años con estenosis laríngea por penfigoide cicatricial que también requirió traqueostomia ${ }^{14}$. En resumen, si bien el compromiso laríngeo en el penfigoide cicatricial es de baja frecuencia, se debe sospechar y buscar activamente, ya que incluso puede llegar a comprometer la vía aérea y requerir de traqueostomía para su manejo. 


\section{Tratamiento médico del penfigoide cicatricial}

Dado que se trata de una patología inhabitual, no existen trabajos randomizados que apoyen las medidas terapéuticas que habitualmente se utilizan. Como medidas generales se sugiere higiene oral intensiva, y evitar condimentos ${ }^{5}$.

Corticoides. En el tratamiento farmacológico se utilizan corticoides sistémicos, generalmente prednisona 20-40 mg/día por 5 días y luego 20-10 $\mathrm{mg}$ diario por 2 semanas ${ }^{15}$. Este tratamiento puede ser acompañado de corticoides tópicos en las lesiones.

Dapsona Posee propiedades antinflamatorias y se cree que inhibe la adherencia de neutrófilos e inhibiría la síntesis de prostaglandina E2. Para su uso se debe descartar la presencia de un déficit de la enzima glucosa 6-fosfato deshidrogenasa, ya que puede producir anemia hemolítica. Para minimizar los efectos adversos, se sugiere utilizar un régimen de dapsona de $25 \mathrm{mg}$ por tres días, aumentando de $25 \mathrm{mg}$ cada tres días hasta llegar a $150 \mathrm{mg}$ diarios $^{5}$.

Aros. La azatioprina (1-2 mg/Kg día) y la ciclofosfamida (0,5-2 mg/Kg día) Son utilizadas en casos severos en conjunto con corticoides. Existen reportes del uso de metotrexato, leflunomida, talidomida, tetraciclina y doxiciclina, pero se necesitan de estudios randomizados para valorar el uso de estos fármacos en el tratamiento del penfigoide cicatricial ${ }^{5}$.

\section{CONCLUSIONES}

El penfigoide cicatricial es una enfermedad de baja frecuencia, que afecta principalmente a las mucosas oral y ocular. La mucosa de la laringe sólo se compromete en $10 \%$ de estos pacientes, pudiendo incluso a producir una estenosis supraglótica que requiera de traqueostomía. Frente al diagnóstico diferencial de una estenosis supraglótica benigna se debe tener presente al penfigoide cicatricial.

\section{REFERENCIAS}

1. Milián-Masanet Ma, Sanchis-Bielsa JM. Penfigoides: Revisión y puesta al día. ROOE 2004; 9(4): 429-34.

2. Tricamo M, Rees T, Hallmon W, Wright J, Cueva M, Plemons J. Periodontal Status in Patients With Gingival Mucous Membrane Pemphigoid. J Periodontol 2006; 77: 398-405.

3. COOPER W. Pemphigus of the conjunctiva. Ophthalmol Hosp Rep 1857; 1: 155-7.

4. Boedeker C, Staats R, Termeer C, Ridder G. Cicatricial Pemphigoid in the Upper Aerodigestive Tract: Diagnosis and Management in Severe Laryngral Stenosis. Ann Otol Rhinol Laryngol 2003; 112: 271-5.

5. Scully C, MuzıoB LL. Oral mucosal diseases: Mucous membrane pemphigoid. British Journal of Oral and Maxillofacial Surgery 2008; 46: 358-66.

6. Darling M, Daley T. Blistering Mucocutaneous Diseases of the Oral Mucosa - A Review: Part 1. Mucous Membrane Pemphigoid. J Can Dent Assoc 2005; 71(11): 851-4.

7. Chan L, Ahmed AR, Anhalt G, Bernauer W, Cooper K, Elder M et AL. The First International Consensus on Mucous Membrane Pemphigoid. Arch Dermatol 2002; 138: 370-9.

8. Dayan S, Simmons R, Ahmed AR. Contemporary issues in the diagnosis of oral pemphigoid. Oral Surg Oral Med Oral Pathol Oral Radiol Endod 1999; 88: 424-30.

9. ISENBERG S. Esophageal presentation of cicatrical pemphigoid. Aolaryngology - Head and Neck Surgery 1998; 118(6): 845-7.

10. Challacombe SJ, Setterfield J, Shirlaw P, Harman K, Scully C, BLACK MM. Immunodiagnosis of pemphigus and mucous membrane pemphigoid. Acta Odontol Scand 2001; 59(4): 226-34.

11. Tновт A. Der chronische Schleimhautpemphigus der oberen Luftwege. Arch Laryngol Rhinol 1911; 25: 459-78.

12. Hanson RD, OLsen KD, Rogers RS $3^{\text {RD. Upper }}$ aerodigestive tract manifestations of cicatricial pemphigoid. Ann Otol Rhinol Laryngol 1988; 97: 493-9.

13. Gaspar ZS, WoJnarowska F. Cicatricial pemphigoid with severe laryngeal involvement necessitating tracheostomy (laryngeal cicatricial pemphigoid). Cin Exp Dermatol 1996; 21: 209-10. 
14. Ojha J, Bhattacharyya I, Stewart C, Katz J. Cicatricial pemphigoid with severe gingival and laryngeal involvement in an 18-year-old female. Oral Surg Oral Med Oral Pathol Oral Radiol Endod 2007; 104: 363-7.
15. Vincent SD, Lilly Ge, Baker KA. Clinical, historic, and therapeutic features of cicatricial pemphigoid. A literature review and open therapeutic trial with corticosteroids. Oral Surg Oral Med Oral Pathol 1993; 76: 453-9.

Dirección: Dr. Héctor Bahamonde S.

Servicio Otorrinolaringología, Hospital Clínico de la Universidad de Chile. Independencia, Santiago, Chile.

Santos Dumont 999. Teléfono: 9788153. 